\title{
Functional electrospun polymeric nanofibers incorporating geraniol-cyclodextrin inclusion complexes: High thermal stability and enhanced durability of geraniol
}

\author{
Fatma Kayaci ${ }^{\mathrm{a}, \mathrm{b}}$, H. Sener Sen ${ }^{\mathrm{a}}$, Engin Durgun ${ }^{\mathrm{a}, \mathrm{b}}$, Tamer Uyar ${ }^{\mathrm{a}, \mathrm{b}, *}$ \\ a UNAM-National Nanotechnology Research Center, Bilkent University, Ankara 06800, Turkey \\ b Institute of Materials Science E' Nanotechnology, Bilkent University, Ankara 06800, Turkey
}

\section{A R T I C L E I N F O}

\section{Article history:}

Received 11 July 2013

Accepted 29 March 2014

Available online 5 April 2014

\section{Keywords:}

Cyclodextrin

Geraniol

Inclusion complex

Electrospinning

Nanofiber

Polyvinyl alcohol (PVA)

\begin{abstract}
A B S T R A C T
In this study, solid geraniol/cyclodextrin inclusion complexes (geraniol/CD-IC) were successfully prepared by using three types of native $C D(\alpha-C D, \beta-C D$ and $\gamma-C D)$. The modeling studies for inclusion complexation between $\mathrm{CD}$ and geraniol were performed by using ab initio techniques. Both experimentally and theoretically, the complexation efficiency between geraniol and $\gamma$-CD was higher; therefore, geraniol $/ \gamma$ - $C D$-IC was chosen and then incorporated into polyvinyl alcohol (PVA) nanofibers (NF) via electrospinning. The scanning electron microscopy imaging elucidated that the aggregates of geraniol $/ \gamma$-CD-IC crystals were distributed in the PVA NF, whereas bead-free and uniform PVA and PVA/geraniol NF without CD-IC were obtained. Higher thermal stability of geraniol was observed in the electrospun PVA/geraniol $/ \gamma$-CD-IC NF. However, geraniol molecules having volatile nature could not be preserved without CD-IC during electrospinning or during storage; therefore, the complete evaporation of geraniol in PVA/geraniol NF was unavoidable even after one day of its production. On the contrary, the loss of geraniol was minimal ( 10\%) for PVA/geraniol/ $\gamma$-CD-IC NF even after storage of these NF for two years owing to inclusion complexation. Our study demonstrated that electrospun NF incorporating CD-IC may be quite applicable in food industry, e.g.: active food packaging or functional foods, due to very high surface area and nanoporous structure of NF; high thermal stability and enhanced durability of active agents and functional food ingredients.
\end{abstract}

(c) 2014 Elsevier Ltd. All rights reserved.

\section{Introduction}

Geraniol, a natural component of plant essential oils, having roselike odor and taste is generally used as a fragrance/flavor in food industry to treat infectious diseases and/or preserve the food (SolórzanoSantos \& Miranda-Novales, 2012); therefore, geraniol presents several remarkable properties such as insect repellent, antimicrobial, antioxidant, anti-inflammatory and anticancer (Chen \& Viljoen, 2010). It is acknowledged as generally-recognized-as-safe by Food and Drug Administration (Lapczynski, Bhatia, Foxenberg, Letizia, \& Api, 2008). However sustainability of geraniol is a major challenge because of its volatile nature.

Molecular encapsulation of volatile or unstable food additives by using cyclodextrins $(\mathrm{CD})$ has been commonly used to extend the product shelf-life by improving stability of these additives (Ciobanu, Landy, \& Fourmentin, 2013; de Vos, Faas, Spasojevic, \& Sikkema, 2010). CD

\footnotetext{
* Corresponding author at: Institute of Materials Science \& Nanotechnology, Bilkent University, Ankara 06800, Turkey. Tel.: + 90 3122903571; fax: + 903122664365.

E-mail address: tamer@unam.bilkent.edu.tr (T. Uyar).
}

are cyclic oligosaccharides having truncated cone shaped molecular structures, and they have capability of forming non-covalent hostguest inclusion complexes (IC) with a variety of molecules such as antibacterials, antioxidants, fragrances/flavors and essential oils (Del Valle, 2004; Hedges, 1998; Marques, 2010; Szejtli, 1998). When the guest molecules encapsulated in CD cavities, they can be protected from evaporation, degradation and oxidation, and moreover controlled/delayed release of these molecules is achieved (Del Valle, 2004; Hedges, 1998; Kant, Linforth, Hort, \& Taylor, 2004; Kayaci \& Uyar, 2011; Marques, 2010; Samperio, Boyer, Eigel, Holland, McKinney, O'Keefe, et al., 2010; Szejtli, 1998). Thus, the enhanced viability of the complexed molecules is provided in CD-IC systems. The most common CD types are $\alpha-, \beta-$, and $\gamma$-CD having $\alpha$-1,4-linked 6, 7, and 8 glucopyranose units in the cyclic structure, and $\sim 6,8$, and $10 \AA$ of cavity size, respectively; whereas the depth of the cavity for these $\mathrm{CD}$ is the same which $\sim 8 \AA$ is (Fig. 1a) (Szejtli, 1998).

Nowadays, electrospinning has gained great attention as the most versatile and cost-effective technique for producing functional nanofibers (NF) that have several unique properties such as a relatively large surface area to volume ratio, nanoporous structure and high encapsulation efficiency (Ramakrishna, 2005; Wendorff, Agarwal, \& Greiner, 

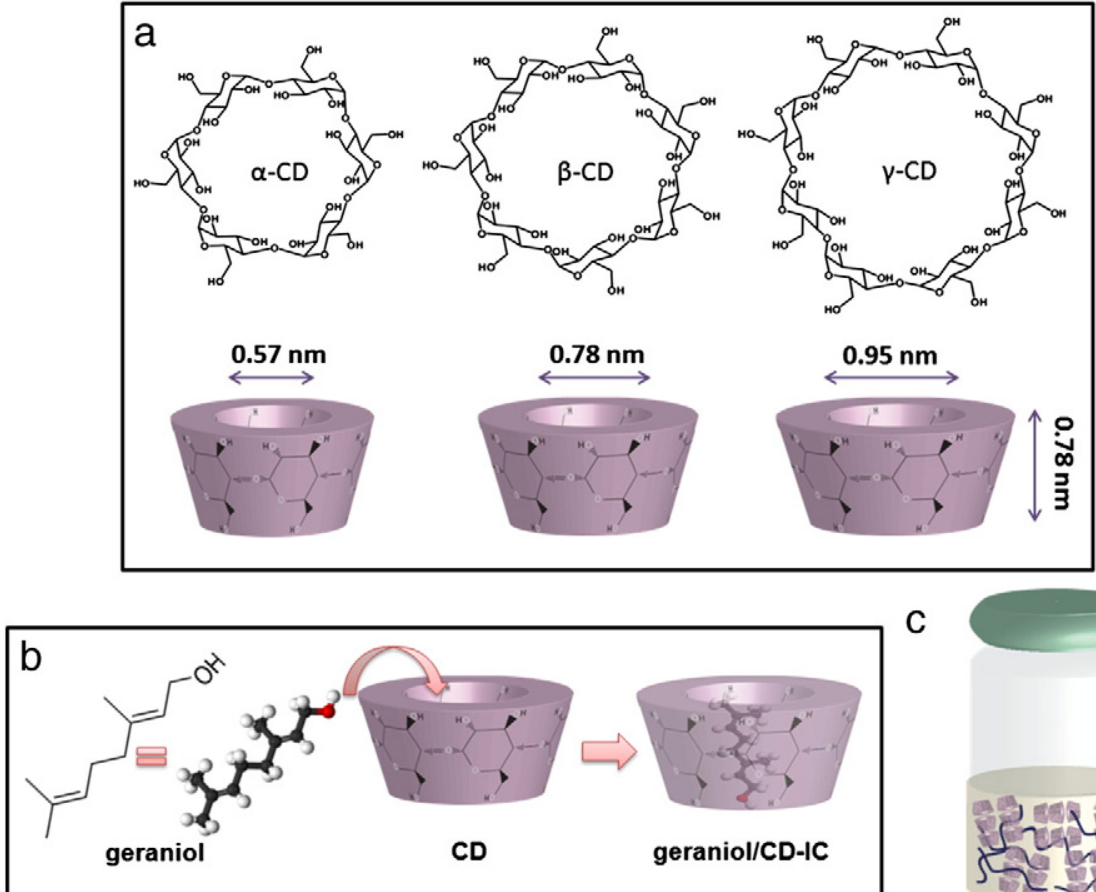

C
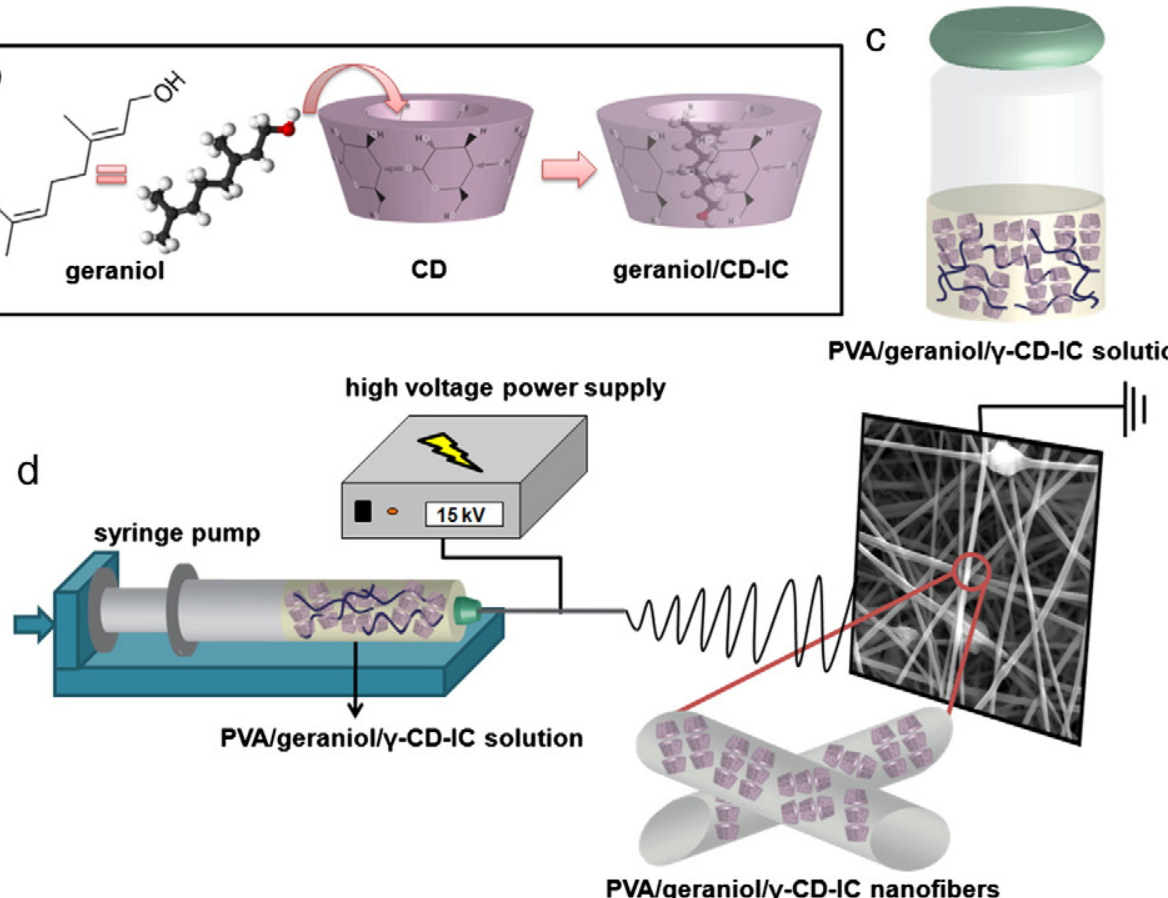

PVA/geraniol/Y-CD-IC nanofibers

e

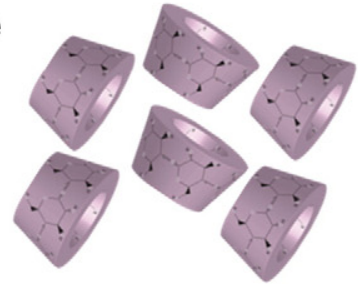

f

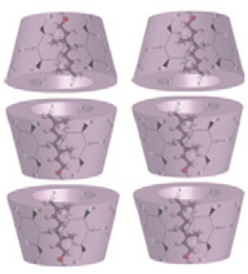

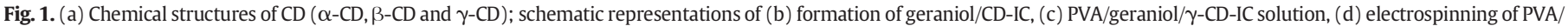
geraniol $/ \gamma$-CD-IC solution, (d) cage-type and (e) channel-type of $\gamma$-CD crystals.

2012). Moreover, the properties of electrospun NF obtained from wide range of materials such as various synthetic or natural polymers, polymer blends, sol-gels, ceramics, and composites, can be improved by incorporating functional additives into the fiber matrix and/or onto fiber surface (Ramakrishna, 2005; Wendorff et al., 2012). Therefore, electrospun NF are favorable candidates in various application areas including filtration, controlled/sustained release systems, drug delivery, tissue engineering, wound healing, functional textiles, energy and environment (Ramakrishna, 2005; Wendorff et al., 2012). Electrospun NF from biodegradable (Ignatova, Manolova, \& Rashkov, 2013; TorresGiner, Gimenez, \& Lagaron, 2008) or edible (Mascheroni, Fuenmayor,
Cosio, Di Silvestro, Piergiovanni, Mannino, et al., 2013) polymers has also received great attention in functional food and active food packaging systems, recently. Moreover, food related applications and food packaging application were investigated for electrospun NF based on food grade materials (Kuan, Yuen, Bhat, \& Liong, 2011; Stijnman, Bodnar, \& Hans Tromp, 2011) and/or NF incorporating active agents such as antibacterials (Ge, Zhao, Mo, Li, \& Li, 2012; Kriegel, Kit, McClements, \& Weiss, 2008; Li, Lim, \& Kakuda, 2009; Vega-Lugo \& Lim, 2009), antioxidants (Fernandez, Torres-Giner, \& Lagaron, 2009; Neo, Ray, Jin, Gizdavic-Nikolaidis, Nieuwoudt, Liu, et al., 2012) or essential oils (Kriegel et al., 2008). Development of nanocomposite fabrics 
with the functional electrospun NF that can supply antimicrobial protection or delivery of nutraceuticals to foods could improve the performance for bioactive packaging applications (Torres-Giner, 2011).

Our particular interest is the functionalization of electrospun $\mathrm{NF}$ with CD-IC, and hereby the combining of unique properties of both $\mathrm{NF}$ and CD-IC. We have recently incorporated menthol/CD-IC (Uyar, Hacaloglu, \& Besenbacher, 2009, 2011; Uyar, Nur, Hacaloglu, \& Besenbacher, 2009), vanillin/CD-IC (Kayaci \& Uyar, 2012), eugenol/ CD-IC (Kayaci, Ertas, \& Uyar, 2013) and triclosan/CD-IC (Kayaci, Umu, Tekinay, \& Uyar, 2013) in the polymeric NF via electrospinning. Prolonged shelf-life and high thermal stability of menthol, vanillin or eugenol in the resulting NF were observed by means of inclusion complexation. The NF incorporating triclosan/CD-IC have shown better antibacterial property compared to the system of without CD-IC possibly due to the enhanced solubility and effective release of triclosan into agar medium by CD-IC (Kayaci et al., 2013).

In this study, as a first step we prepared solid CD-IC of geraniol (geraniol/CD-IC) by using $\alpha-C D, \beta-C D$, and $\gamma-C D$. We have also performed the modeling studies for inclusion complexation between geraniol and $\mathrm{CD}(\alpha-\mathrm{CD}, \beta-\mathrm{CD}$, and $\gamma-\mathrm{CD})$ by using ab initio techniques. Both experimentally and theoretically, we observed that $\gamma$-CD has shown higher complexation efficiency with geraniol, yielding 1:1 molar ratio (geraniol: $\gamma$-CD) of solid CD-IC crystals. Then, geraniol $/ \gamma$-CD-IC was encapsulated in polyvinyl alcohol (PVA) NF via electrospinning in order to enhance thermal stability and durability of geraniol in the NF. Fig. 1b and $\mathrm{c}$ indicated the schematic representations of the formation of geraniol/CD-IC and electrospinning of PVA solution including geraniol/ $\gamma$-CD-IC (PVA/geraniol/ $\gamma$-CD-IC), respectively. Here, the reason of the selection of PVA as a polymeric matrix is to be a proper polymer type in food packaging application due to its biodegradability (DeMerlis \& Schoneker, 2003). We observed that PVA NF could not preserve geraniol without the CD-IC, yet incorporation of geraniol $/ \gamma-C D-I C$ in PVA NF successfully yielded geraniol with long lasting durability and enhanced thermal stability, hence the resulting functional PVA electrospun NF incorporating geraniol $/ \gamma$-CD-IC can be a quite appropriate material for functional food packaging.

\section{Materials and methods}

\subsection{Materials}

Cyclodextrins ( $\alpha-C D, \beta-C D$, and $\gamma-C D$, Wacker Chemie AG), geraniol (trans-3,7-dimethyl-2,6-octadien-1-ol, 98\% purity, Sigma-Aldrich), polyvinyl alcohol (PVA, $\mathrm{M}_{\mathrm{w}}$ : 85,000-124,000, 87-89\% hydrolyzed, Sigma-Aldrich) and deuterated dimethylsulfoxide (DMSO-d6, deuteration degree min. 99.8\% for NMR spectroscopy, Merck) were used in this study without any purification. The water used as solvent was obtained from Millipore Milli-Q ultrapure water system.

\subsection{Preparation of solid geraniol/CD-IC}

The geraniol/CD-IC were prepared by co-precipitation method by using $\alpha-C D, \beta-C D$, and $\gamma-C D$. Equimolar ratio (1:1) of geraniol with $C D$ was used. First, $0.159 \mathrm{~g}\left(1.0 \times 10^{-3} \mathrm{~mol}\right), 0.136 \mathrm{~g}\left(8.8 \times 10^{-4} \mathrm{~mol}\right)$ and $0.119 \mathrm{~g}\left(7.7 \times 10^{-4} \mathrm{~mol}\right)$ geraniol were dispersed in water, and then $1 \mathrm{~g}$ of $\alpha-C D\left(1.0 \times 10^{-3} \mathrm{~mol}\right), \beta-C D\left(8.8 \times 10^{-4} \mathrm{~mol}\right)$, and $\gamma-C D$ $\left(7.7 \times 10^{-4} \mathrm{~mol}\right)$ were added in these geraniol dispersions, respectively. The amount of water used as solvent was determined according to the solubility of $\mathrm{CD}$ in water at $25^{\circ} \mathrm{C}$, that is, $14.5,1.85$ and $23.2 \mathrm{~g} / 100 \mathrm{~mL}$ for $\alpha-C D, \beta-C D$ and $\gamma$-CD, respectively (Del Valle, 2004; Szejtli, 1998). After mixing the solutions overnight at room temperature (RT), the resulting three suspensions were filtered through a borosilicate filter (por. 3). Thereafter, the filtrates were washed with water several times in order to remove uncomplexed molecules if any present, and then dried overnight under the hood. Lastly, the resulting solid geraniol/CD-IC were crushed in a mortar to obtain fine powder.

\subsection{Preparation of the electrospinning solutions}

Among the three $\mathrm{CD}$ types, we observed that $\gamma$-CD has higher complexation efficiency with geraniol under the chosen experimental conditions; hence, we have selected geraniol $/ \gamma$-CD-IC to incorporate into PVA NF via electrospinning technique. Firstly, $12 \%$ (w/v) PVA was dissolved in water by stirring for $2 \mathrm{~h}$ at $80{ }^{\circ} \mathrm{C}$, and the solution was left at $\mathrm{RT}$ to cool down. After that, geraniol $/ \boldsymbol{\gamma}$-CD-IC fine powder was dispersed in this polymer solution, and then the solution was stirred for additional $5 \mathrm{~h}$ at RT. The amount of geraniol/ $\gamma$-CD-IC was adjusted as $50 \%(w / w)$, so as to include $5 \%(\mathrm{w} / \mathrm{w})$ geraniol in the solution as regards the PVA content. For a comparison study, PVA and PVA/geraniol solutions without CD-IC were also prepared to obtain PVA and PVA/geraniol NF. Table 1 summarizes the compositions of the solutions used for electrospinning.

\subsection{Electrospinning}

Each prepared solution was located in a $5 \mathrm{~mL}$ syringe having a metallic needle of $0.7 \mathrm{~mm}$ inner diameter, and the syringe was placed horizontally on the syringe pump (KD Scientific, KDS 101). The solutions were pumped with flow rate of $1 \mathrm{ml} / \mathrm{h}$, and tip-to-collector distance was set to $10 \mathrm{~cm}$. When voltage of $15 \mathrm{kV}$ was applied by using high voltage power supply (Matsusada Precision, AU Series) to the metal needle, the NF were deposited on the grounded stationary cylindrical metal collector (height: $15 \mathrm{~cm}$, diameter: $9 \mathrm{~cm}$ ) covered with a piece of aluminum foil. The electrospinning was carried out at about $22{ }^{\circ} \mathrm{C}$ and $25 \%$ relative humidity in enclosed Plexiglas box. Then, the resulting NF were kept in the suction hood at RT for $24 \mathrm{~h}$ to remove the residual solvent and uncomplexed geraniol if any present.

\subsection{Characterization and measurements}

The crystalline structures of the samples were investigated by X-ray diffraction (XRD, PANalyticalX'Pert Powder diffractometer) with $\mathrm{Cu} \mathrm{K \alpha}$ radiation in a range of $2 \theta=5^{\circ}-30^{\circ}$. Thermogravimetric analyzer (TGA, Q500, TA Instruments) was used to investigate the thermal stability of geraniol, and the analyses were performed from RT to $250{ }^{\circ} \mathrm{C}$ (geraniol), $500{ }^{\circ} \mathrm{C}\left(\gamma\right.$-CD and geraniol/CD-IC) or $600{ }^{\circ} \mathrm{C}$ (electrospun NF) at a $20{ }^{\circ} \mathrm{C} / \mathrm{min}$ heating rate, with purge gas of $\mathrm{N}_{2}$. About $20 \mathrm{~g} / \mathrm{L}$ of the samples were dissolved in DMSO- $d 6$ for proton nuclear magnetic resonance (1H- NMR, DPX-400, Bruker) study, and then $1 \mathrm{H}$ - NMR spectra were recorded at $400 \mathrm{MHz}$ and $25{ }^{\circ} \mathrm{C}$. Rheometer (Physica MCR 301, Anton Paar) equipped with a cone/plate accessory (spindle type: $\mathrm{CP} 40-2$ ) was used to measure the viscosity of the solutions used for electrospinning, at a constant shear rate of $100 \mathrm{~s}^{-1}$ and $22^{\circ} \mathrm{C}$. Scanning electron microscope (SEM, Quanta 200 FEG, FEI) was used to analyze the morphology and fiber diameter of the electrospun NF that were sputtered with $5 \mathrm{~nm} \mathrm{Au} / \mathrm{Pd}$ (PECS-682) prior to SEM imaging. Average fiber diameter (AFD) of the samples was calculated by measuring around 100 fiber diameters of each sample.

\subsection{Computational method}

The first-principle calculations based on density functional theory (DFT) (Hohenberg \& Kohn, 1964; Kohn \& Sham, 1965) were performed by using the Vienna Ab initio simulation package (Kresse \& Furthmüller, 1996a, 1996b). The exchange-correlation was approximated within the generalized gradient approximation (Perdew, Chevary, Vosko, Jackson, Pederson, Singh, et al., 1992) with addition of Van der Waals correction (Grimme, 2006). The element potentials were described by projector augmented-wave method (PAW) (Blöchl, 1994) using a plane-wave basis set with a kinetic energy cutoff of $500 \mathrm{eV}$. The initial structures of $\alpha$-CD (Manor \& Saenger, 1974; Puliti, Mattia, \& Paduano, 1998), $\beta$ CD (Lindner \& Saenger, 1982), and $\gamma$-CD (Harata, 1987) were taken from Cambridge Structural Database (Allen, 2002). All structures were 
Table 1

The properties of the solutions used for electrospinning and morphological characteristics of the electrospun NF.

\begin{tabular}{|c|c|c|c|c|c|c|}
\hline Solutions & $\begin{array}{l}\% \text { PVA }^{a} \\
(\mathrm{w} / \mathrm{v})\end{array}$ & $\begin{array}{l}\text { \% geraniol/CD-IC } \\
(\mathrm{w} / \mathrm{w})\end{array}$ & $\begin{array}{l}\text { \% geraniol } \\
(\mathrm{w} / \mathrm{w})\end{array}$ & Viscosity $(\mathrm{Pa} \cdot \mathrm{s})$ & $\begin{array}{l}\text { Average fiber } \\
\text { diameter (nm) }\end{array}$ & Fiber morphology \\
\hline PVA & 12 & - & - & 0.237 & $195 \pm 50$ & Bead-free NF \\
\hline PVA/geraniol & 12 & - & 5 & 0.264 & $200 \pm 40$ & Bead-free NF \\
\hline PVA/geraniol/ $\gamma-C D-I C$ & 12 & 50 & 5 & 0.372 & $290 \pm 70$ & NF with CD-IC crystals \\
\hline
\end{tabular}

a With respect to the solvent (water)

b With respect to the polymer (PVA).

relaxed using the Kosugi algorithm with simultaneous minimization of the total energy and interatomic forces. The convergence on the total energy and force was set to $10^{-5} \mathrm{eV}$ and $10^{-2} \mathrm{eV} / \mathrm{A}$, respectively.

\section{Results and discussion}

\subsection{Crystalline structure of geraniol/CD-IC}

The co-precipitation method was applied to prepare the solid geraniol/CD-IC by using three types of native $C D(\alpha-C D, \beta-C D$ and $\gamma-$ $\mathrm{CD})$. Initially, the crystalline structures of the solid geraniol/CD-IC were investigated by XRD. The as-received $C D$ were also analyzed by XRD for comparison. The characteristic diffraction patterns of asreceived CD (Fig. 2a) correspond to cage-type packing (Fig. 1d) (Rusa, Bullions, Fox, Porbeni, Wang and Tonelli, 2002; Saenger et al., 1998). The channel-type packing (Fig. 1e) in which CD molecules are aligned and stacked on top of each other, is generally observed for the inclusion complexes, and therefore the presence of channel-type packing is a strong evidence for the successful CD-IC formation (Rusa et al., 2002; Saenger et al., 1998). Here, we observed that the XRD patterns of geraniol/CD-IC (Fig. 2b) are very different from that of as-received CD. The distinct peak at $2 \theta \cong 19.7^{\circ}$ in the XRD pattern of geraniol $/ \alpha-C D-I C$ confirmed the channel-type packing of $\alpha$-CD in this sample (Kayaci \& Uyar, 2011; Rusa et al., 2002). In the case of geraniol/B-CD-IC, the XRD peaks at $2 \theta \cong 12^{\circ}$ and $2 \theta \cong 17.7^{\circ}$ were owing to channel-type packing of $\beta$-CD (Kayaci \& Uyar, 2011). Furthermore, the characteristic peaks of tetragonal channel-type packing of $\gamma$-CD at $2 \theta \cong 7.6^{\circ}, 14^{\circ}, 15^{\circ}$, $15.9^{\circ}, 16.7^{\circ}$ and $21.9^{\circ}$ were observed in the XRD pattern of geraniol $/ \gamma$ CD-IC (Rusa et al., 2002; Uyar, Hunt, Gracz, \& Tonelli, 2006). As a result, channel-type packing of $\alpha-C D, \beta-C D$, and $\gamma-C D$ that were observed in the XRD patterns of solid geraniol/CD-IC confirmed that the inclusion complexation of geraniol with each type of CD was successful.

\subsection{Thermal properties of geraniol/CD-IC}

The thermal stability of geraniol in geraniol/CD-IC was investigated by TGA. The TGA thermograms of $\mathrm{CD}(\gamma-\mathrm{CD})$ and pure geraniol were also given for comparison (Fig. 3a). The TGA data of $\alpha-C D$ and $\beta-C D$ were not given, since the thermograms were quite similar to that of $\gamma$-CD. In TGA thermogram of $\gamma$-CD, initial weight loss at below $100{ }^{\circ} \mathrm{C}$ was due to water loss, and the major weight loss at around $300{ }^{\circ} \mathrm{C}$ corresponded to degradation of $\gamma-\mathrm{CD}$. On the other hand, evaporation of pure geraniol was in the range of $70-230{ }^{\circ} \mathrm{C}$ as observed in its TGA thermogram. In the case of TGA thermogram of geraniol/CD-IC (Fig. 3a), three steps of weigh losses were observed. That is, the initial water loss at below $100{ }^{\circ} \mathrm{C}$ and the main degradation of $\mathrm{CD}$ at $300{ }^{\circ} \mathrm{C}$ were recorded, additionally, the evaporation of geraniol complexed with $\mathrm{CD}$ was observed at above $120{ }^{\circ} \mathrm{C}$ till $300{ }^{\circ} \mathrm{C}$ at which the main degradation of $\mathrm{CD}$ occurred. The evaporation temperature of pure geraniol shifted from $70-230{ }^{\circ} \mathrm{C}$ to $120-300{ }^{\circ} \mathrm{C}$ when complexed with $\mathrm{CD}$ and this was due to the host-guest interaction in the CD-IC samples. The enhanced thermal stabilities of the guest molecules complexed with the CD cavities were also reported for other CD-IC systems (Kayaci \& Uyar, 2011; Marcolino, Zanin, Durrant, Benassi, \& Matioli, 2011; Tsai, Tsai, Wu, \& Tsai, 2010). In brief, the TGA data suggested the inclusion complexation of geraniol with $\alpha-C D, \beta-C D$, and $\gamma-C D$. The evaporation temperature of geraniol in all three CD-IC samples was between 120 and $300{ }^{\circ} \mathrm{C}$, and we did not see any significant difference among the CD-IC samples except for the \% weight loss in this temperature range. It is possible that the evaporation of geraniol could
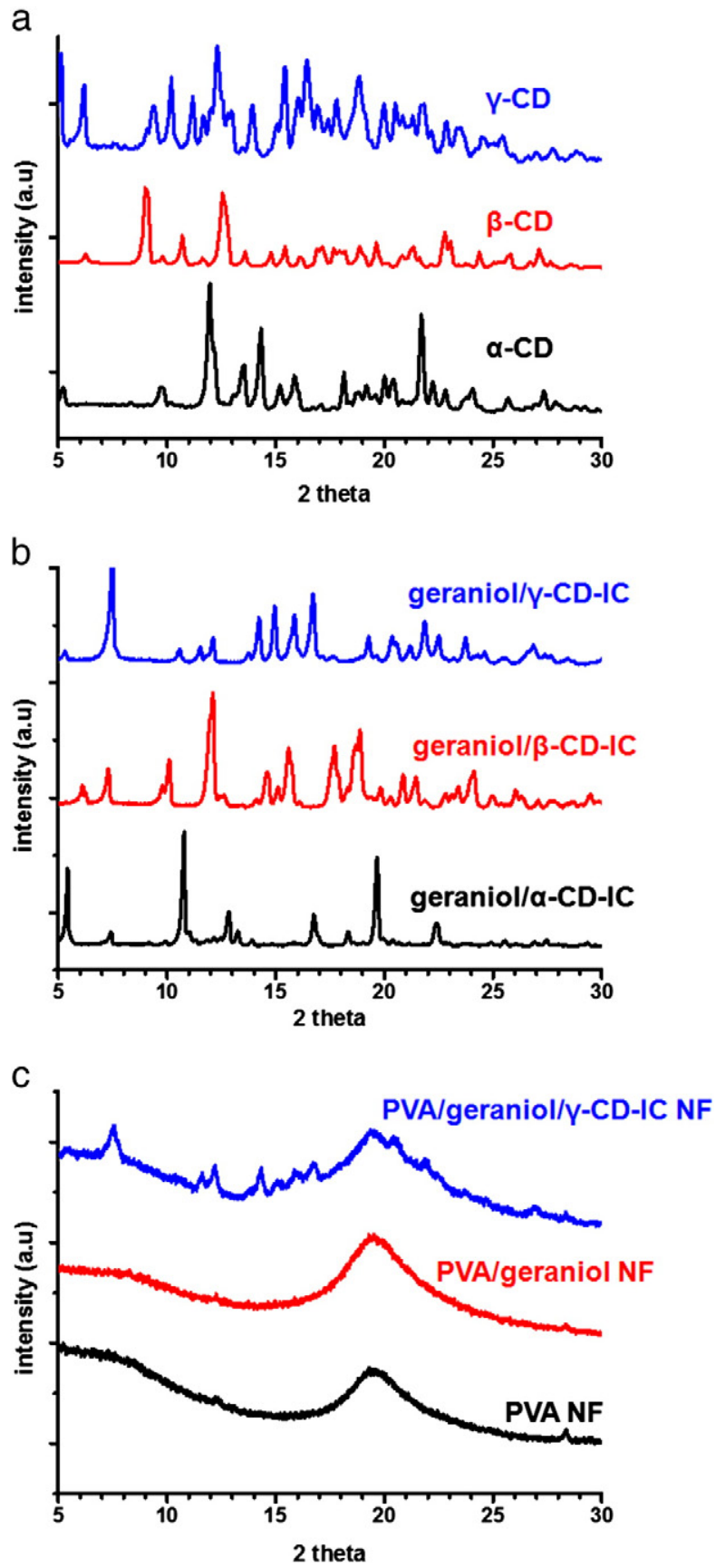

Fig. 2. XRD patterns of (a) as-received CD, (b) geraniol/CD-IC and (c) the electrospun NF. 

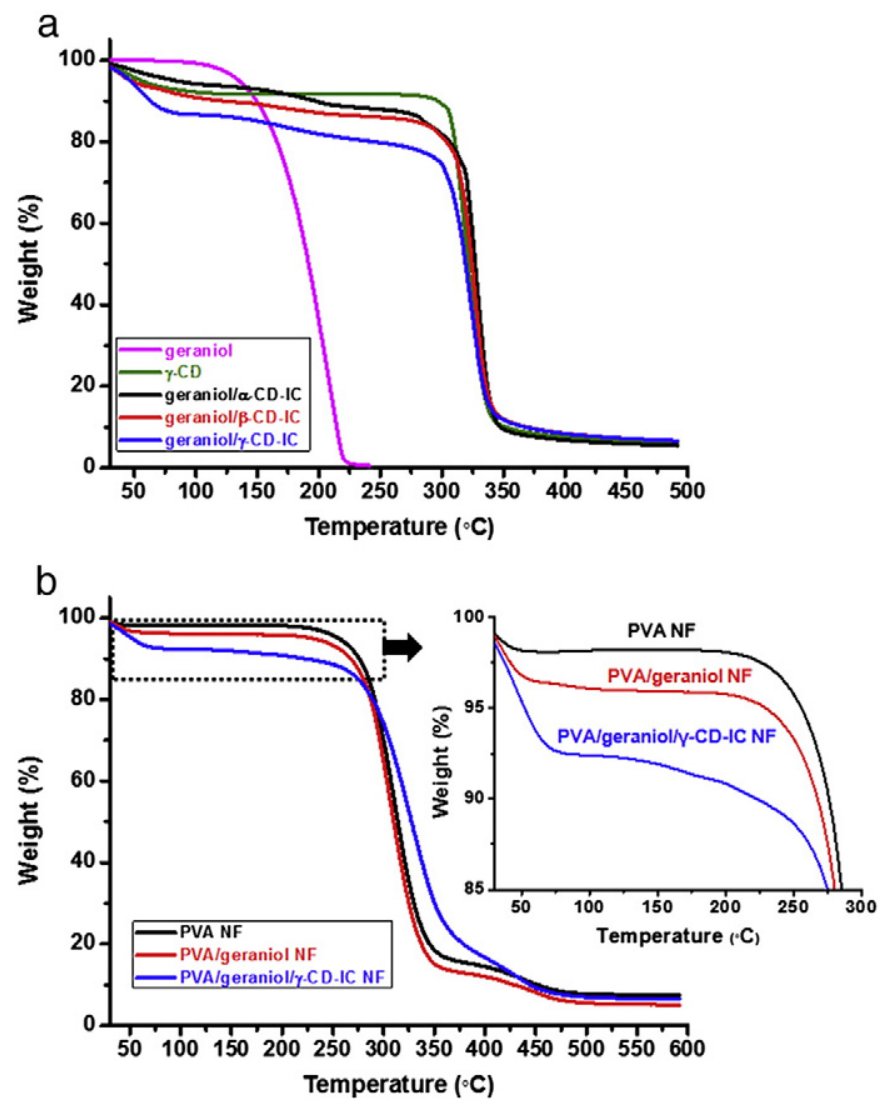

Fig. 3. TGA thermograms of (a) geraniol, $\gamma$-CD, geraniol/CD-IC and (b) the electrospun NF.

continue above $300{ }^{\circ} \mathrm{C}$ where the main degradation of $\mathrm{CD}$ started. So, we did not use TGA thermograms for calculating the actual weight content of geraniol in the CD-IC, instead, this was investigated by ${ }^{1} \mathrm{H} N M R$ study as discussed in the following section.

\subsection{Structural characterization of geraniol/CD-IC}

The structural characterization of geraniol/CD-IC sample was studied by using ${ }^{1} \mathrm{H}$ NMR. Initially, the characteristic peaks correspond to protons of pure $\mathrm{CD}$ and geraniol, were determined from their ${ }^{1} \mathrm{H}$ NMR spectra (data not shown). The three geraniol/CD-IC samples were studied by ${ }^{1} \mathrm{H}$ NMR, and only ${ }^{1} \mathrm{H}$ NMR spectrum of geraniol $/ \gamma$-CD-IC as the representative of geraniol/CD-IC is shown in Fig. 4. All ${ }^{1} \mathrm{H}$ NMR spectra of geraniol/CD-IC indicated characteristic peaks of not only CD but also geraniol, confirming the presence of both geraniol and $\mathrm{CD}$ in these samples. Moreover, ${ }^{1} \mathrm{H}$ NMR spectroscopy is a useful tool for quantitative calculation of guest molecule in CD-IC system. We found out that the molar ratio of geraniol:CD was 0.78:1, 0.9:1 and 1:1 for geraniol/ $\alpha$-CD-IC, geraniol/ $\beta$-CD-IC and geraniol $/ \gamma$-CD-IC, respectively. The calculation was done by the integrations of the $\mathrm{CD}$ peak at about $5.8 \mathrm{ppm}$ $(\mathrm{OH}-2 \& 3)$ and geraniol peak at $5.3 \mathrm{ppm}(\mathrm{H}-\mathrm{b})$. This result indicated that initial molar ratio (geraniol:CD =1:1) used for the preparation of geraniol/CD-IC was only preserved for geraniol $/ \gamma$-CD-IC. However, $\alpha-C D$ and $\beta-C D$ could not complex with all geraniol that was used, and some uncomplexed geraniol was removed from the geraniol $/ \alpha-$ $\mathrm{CD}-\mathrm{IC}$ and geraniol/ $\beta$-CD-IC during sample preparation. As shown in Fig. 1a, the cavity size of $C D$ are different and in the order of $\gamma-C D>$ $\beta-C D>\alpha-C D$. So, the higher complexation efficiency of $\gamma$-CD with geraniol might be due to the larger dimension of $\gamma$-CD cavity which may have a better size match with different conformers of geraniol molecule.
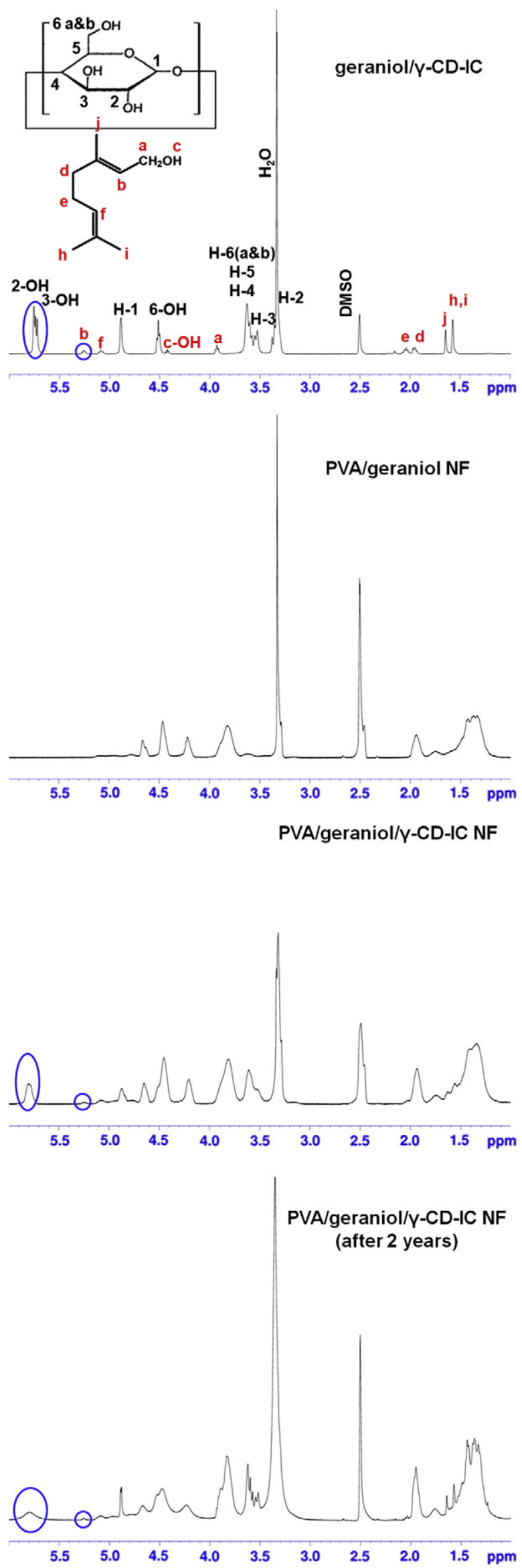

Fig. 4. NMR spectrum of geraniol $/ \gamma$-CD-IC and the electrospun NF. 
Hence, in the next part of this study, geraniol $/ \gamma$-CD-IC was selected and incorporated into the electrospun NF.

\subsection{Computational modeling of geraniol/CD-IC}

The inclusion process between $\mathrm{CD}$ and geraniol is investigated by using ab initio techniques as explained in the Materials and methods section. Firstly, the initial geometries of $\alpha-C D, \beta-C D$, and $\gamma-C D$ and various conformers of geraniol molecule are fully optimized separately in vacuum. In order to form a complex, geraniol molecule with two possible orientations ( $\mathrm{OH}$-end and $\mathrm{CH}_{3}$-end) is introduced into $\mathrm{CD}$ cavity through the wide and narrow rims. In order to analyze the energy variation, the geraniol is initially positioned $6 \AA$ away from the center of the CD cavity, which is chosen as the origin and moved in 1 A steps towards the center (Snor, Liedl, Weiss-Greiler, Viernstein, \& Wolschann, 2009). At each step, the whole system is optimized without imposing any constraints and thus any conformational changes are allowed. For the lowest energy configuration (Fig. 5), the complexation energy for each CD type is calculated as

$\mathrm{E}_{\text {complex }}=\left(E_{\mathrm{CD}}+E_{\text {geraniol }}\right)-E_{\text {geraniol }+\mathrm{CD}}$

where $\mathrm{E}_{\mathrm{CD}}, \mathrm{E}_{\text {geraniol }}$ and $\mathrm{E}_{\text {geraniol }}+\mathrm{CD}$ is the total energy (including van der Waals interaction) of $C D(\alpha-C D, \beta-C D$, and $\gamma-C D)$, geraniol, and geraniol/CD-IC, respectively.

In the case of geraniol $/ \alpha-C D$, the inclusion of geraniol mildly deforms the structure of $\alpha-C D$. This indicates that even the most slender possible conformer of geraniol is large to fit in $\alpha-C D$ cavity. $\mathrm{E}_{\text {complex }}$ is calculated as $16.21 \mathrm{kcal} / \mathrm{mol}$ but in order to fit inside the cavity of $\alpha-\mathrm{CD}$, geraniol has to overcome an energy barrier. Accordingly, geraniol may form a complex at the edge of the wide rim (which is a local energy minimum) instead as shown in Fig. 5a with $10.03 \mathrm{kcal} / \mathrm{mol}$ complexation energy. The $\beta-C D$ and $\gamma-C D$ cavity is large enough to accommodate geraniol without any deformation (Fig. $5 b$ and c). For $\beta-C D$ and $\gamma-\mathrm{CD}, \mathrm{E}_{\text {complex }}$ is 25.83 and $20.75 \mathrm{kcal} / \mathrm{mol}$, respectively and there is no energy barrier for inclusion process. The contribution to complexation energy is mainly due to van der Waals interaction and also extra hydrogen bonds are formed between hydroxyl group of the geraniol and primary hydroxyl group of $\mathrm{CD}$ at the narrow rim. The discussions are for the most slender geraniol conformer only but other conformers can be present as well. For instance, as $\gamma$-CD has the largest cavity it is more flexible to accommodate conformers of geraniol with different sizes (Fig. 5c). a
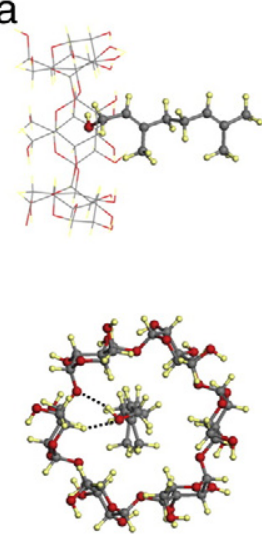

C
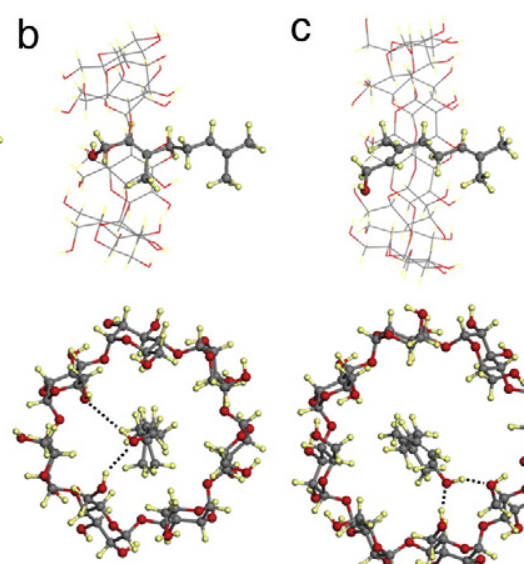

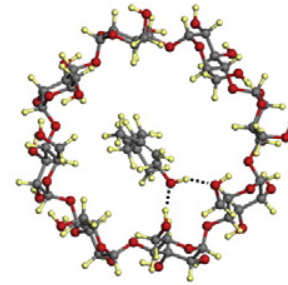

Fig. 5. Side and top view of optimized structures of geraniol a) geraniol/ $\alpha-C D-I C$ b) geraniol $/ \beta-C D-I C$, and c) geraniol $/ \gamma$-CD-IC. Extra hydrogen bonds are shown by dashed lines. Gray, red, and yellow spheres represent carbon, oxygen, and hydrogen atoms, respectively.
Finally, our modeling results show that, even the most slender conformer of geraniol does not fit into $\alpha$-CD yielding relatively low complexation energy. This also indicates the possibility of complex formation outside the cavity. For $\beta-C D$ and $\gamma-C D, E_{\text {complex }}$ is high which is in agreement with our experimental observations. Even though the largest $\mathrm{E}_{\text {complex }}$ is obtained for $\beta-C D$ for the slender geraniol conformer, the large cavity of $\gamma$-CD allows accommodation of various conformers of geraniol and makes it the best candidate for inclusion complexation.

\subsection{The morphological structure of the NF}

In this part of the study, geraniol $/ \gamma$-CD-IC crystals were incorporated in the PVA NF via electrospinning in order to produce functional nanofibrous material. The geraniol $/ \gamma$-CD-IC fine powder was dispersed in the PVA solution, and then this solution was electrospun to encapsulate the geraniol $/ \gamma-C D-I C$ in PVA NF. We also produced electrospun PVA and PVA/geraniol NF without CD-IC under the same electrospinning conditions for comparative study. Fig. 6 indicates the representative SEM images of PVA, PVA/geraniol and PVA/geraniol $/ \gamma$-CD-IC NF. Moreover, Table 1 summarizes the viscosity of the solutions used for the electrospinning and the morphological findings with AFD of the resulting electrospun NF. PVA and PVA/geraniol NF were uniform and bead-free, whereas the aggregates of geraniol $/ \gamma$-CD-IC crystals in PVA/ geraniol $/ \gamma$-CD-IC NF were seen in SEM images in which the presence of CD-IC crystals were also confirmed by the XRD as discussed in the following section. The viscosities of PVA and PVA/geraniol solutions were very similar to each other, hence AFD of the NF obtained from these solutions were almost same. On the other hand, higher viscosity was found for PVA/geraniol/ $\gamma$-CD-IC solution compared to PVA and PVA/geraniol solutions possibly owing to the interaction between the geraniol/ $\gamma$-CD-IC and PVA polymer chains. Therefore, less stretching of the PVA/ geraniol/ $\gamma$-CD-IC solution possibly occurred during electrospinning process due to its higher viscosity; accordingly, higher AFD was observed for PVA/geraniol/ $\gamma$-CD-IC $(290 \pm 70 \mathrm{~nm})$ system when compared to PVA $(195 \pm 50 \mathrm{~nm})$ and PVA/geraniol $(200 \pm 40 \mathrm{~nm})$ systems. This result correlates with the general observation that is obtaining thicker NF from the solution having higher viscosity (Bhardwaj \& Kundu, 2010; Ramakrishna, 2005).

\subsection{The crystalline structure of the NF}

We have investigated the crystalline structure of the resulting NF by using XRD. The XRD patterns of both PVA and PVA/geraniol NF (Fig. 2c) have very similar broad diffraction centered at $2 \theta \cong 20^{\circ}$ due to semicrystalline nature of PVA matrix. The XRD pattern of PVA/geraniol/ $\gamma$-CD-IC NF (Fig. 2c) has also broad diffraction centered at $2 \theta \cong 20^{\circ}$, indicating that the presence of geraniol $/ \gamma$-CD-IC did not affect the semi-crystalline nature of the polymeric matrix. Furthermore, the characteristic diffraction peaks of channel-type packing of $\gamma$-CD observed in the XRD pattern of PVA/geraniol $/ \gamma$-CD-IC NF have demonstrated the existence of geraniol $/ \gamma$-CD-IC crystals in this sample (Fig. $2 c$ ). It was deduced that channel-type packing of geraniol $/ \gamma$-CD-IC were conserved during the solution preparation and electrospinning process, and then these crystalline aggregates of geraniol $/ \gamma$-CD-IC were successfully encapsulated within the PVA NF matrix, as it was also clearly observed in the SEM image of PVA/geraniol/ $\gamma$-CD-IC NF (Fig. 6c).

\subsection{The thermal properties of the NF}

TGA thermograms of PVA, PVA/geraniol and PVA/geraniol $/ \gamma$-CD-IC $\mathrm{NF}$ are given in Fig. 3b. The major weight loss of TGA thermogram of PVA NF started at about $250{ }^{\circ} \mathrm{C}$ owing to thermal degradation of PVA. The geraniol could not be detected in TGA thermogram of PVA/geraniol $\mathrm{NF}$, since no difference was observed between the TGA thermograms of PVA and PVA/geraniol NF. This result indicated that the loss of geraniol possibly took place during the electrospinning of PVA/geraniol solution 

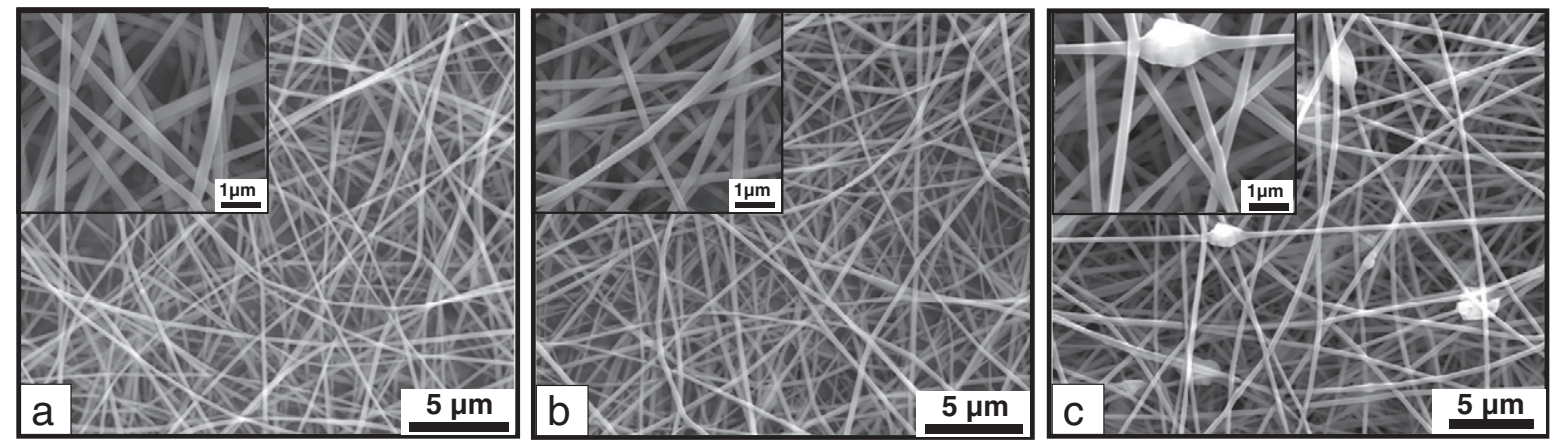

Fig. 6. Representative SEM images of (a) PVA, (b) PVA/geraniol and (c) PVA/geraniol $/ \gamma$-CD-IC NF.

or storage of these NF in suction hood at RT for $24 \mathrm{~h}$, and eventually; volatile geraniol could not be preserved without CD-IC. In the case of PVA/ geraniol $/ \gamma$-CD-IC NF, the evaporation of complexed geraniol was observed at between 120 and $250{ }^{\circ} \mathrm{C}$ till the degradation of PVA starts. As a result, TGA data further confirmed the existence of geraniol/ $\gamma$-CD-IC in PVA NF matrix. The calculation of the quantity of geraniol in the NF samples was not done from TGA data, since there might be overlapping of degradation temperature of PVA and $\gamma$-CD with the evaporation of complexed geraniol. So, we carried out ${ }^{1} \mathrm{H}$ NMR study in order to calculate the actual content of remaining geraniol in the $\mathrm{NF}$, and this data is discussed in the following section.

\subsection{Investigation of geraniol content in the NF}

We performed ${ }^{1} \mathrm{H}$ NMR study to calculate the amount of geraniol in $\mathrm{PVA} /$ geraniol$/ \boldsymbol{\gamma}$-CD-IC NF (Fig. 4). The $\boldsymbol{\gamma}$-CD peak at $5.8 \mathrm{ppm}(\mathrm{OH}-2 \& 3)$ and geraniol peak at $5.3 \mathrm{ppm}(\mathrm{H}-\mathrm{b})$ which are not overlapped with the characteristic peaks of PVA were used to calculate the molar ratio of geraniol:CD in PVA/geraniol/ $\gamma$-CD-IC NF sample. The molar ratio of geraniol to $\gamma$-CD was detected as 1:1 which is the same ratio of gerani$\mathrm{ol}: \mathrm{CD}$ in geraniol $/ \gamma$-CD-IC. It was assumed that the initial amount of geraniol in the PVA/geraniol $/ \gamma$-CD-IC solution was preserved during the electrospinning process by means of inclusion complexation. The characteristic peak of geraniol at about $5.3 \mathrm{ppm}(\mathrm{H}-\mathrm{b})$ was not observed in the case of ${ }^{1} \mathrm{H}$ NMR spectrum of PVA/geraniol NF. This result correlated with TGA data, confirmed that all geraniol was evaporated from this sample during either the electrospinning process or storage of the NF in the suction hood for $24 \mathrm{~h}$ after production. So, it was obvious that PVA NF could not protect geraniol molecules without CD-IC.

We have also performed the durability test for PVA/geraniol $/ \gamma$-CDIC NF sample with the open air experiments to show the durability of geraniol in the electrospun NF sample despite the volatile nature of geraniol. The presence of geraniol quantity in the PVA/geraniol/ $\gamma$-CD-IC $\mathrm{NF}$ after two years of their storage in the open air in the laboratory (at about $22{ }^{\circ} \mathrm{C}$ and $25 \%$ relative humidity) was determined by ${ }^{1} \mathrm{H}$ NMR study. The geraniol peak was still present, and it was even observed that the molar ratio of geraniol: $\gamma$-CD was $0.87: 1$ which was very close to the $1: 1$ initial molar ratio. This result showed that very small amount of geraniol $(\sim 10 \%)$ has evaporated from the PVA/geraniol/ $\gamma$-CD-IC NF even after two years of its storage. It was evident that the prolonged durability of geraniol in the NF sample was provided by CD inclusion complexation. Hence, PVA/geraniol $/ \gamma$-CD-IC NF can be applicable in food packaging application since geraniol has antimicrobial and antioxidant properties and therefore PVA/geraniol/ $\gamma$-CD-IC NF can preserve the food effectively for a long time due to prolonged durability of geraniol.

\section{Conclusions}

As a first step, solid geraniol/CD-IC were obtained using $\alpha-C D, \beta-C D$ and $\gamma$-CD by co-precipitation method. The XRD patterns showed that complexation was achieved for all three types of $\mathrm{CD}$. The TGA data indicated that complexed geraniol with $\mathrm{CD}$ had higher thermal evaporation (about $120-300{ }^{\circ} \mathrm{C}$ ) compared to pure geraniol $\left(70-230{ }^{\circ} \mathrm{C}\right.$ ). Our ab initio modeling results show that, geraniol-CD complexation energy ( $E_{\text {complex }}$ ) is higher for $\beta-C D$ and $\gamma-C D$ compared to $\alpha-C D$. Although the largest $E_{\text {complex }}$ was obtained for $\beta-C D$ for the slender geraniol conformer, the large cavity of $\gamma$-CD allows accommodation of various conformers of geraniol and makes it the best candidate for inclusion complexation. Due to higher complexation efficiency of $\gamma$-CD with geraniol compared to other CD types, geraniol $/ \gamma$-CD-IC was selected and incorporated in the electrospun PVA NF. As a second part of this study, PVA NF incorporating geraniol $/ \gamma$-CD-IC were obtained successfully by electrospinning of PVA solution containing geraniol $/ \gamma$-CD-IC dispersion. Bead-free and uniform NF were obtained for PVA and PVA/geraniol NF samples, whereas the SEM images of PVA/geraniol $/ \gamma$-CD-IC NF showed that the aggregates of geraniol/ $\gamma$-CD-IC crystals were present and encapsulated in PVA NF matrix. Furthermore, the presence of CD-IC crystals in the PVA/geraniol/ $\gamma$-CD-IC NF was also confirmed by the XRD data. The geraniol could not be detected in either TGA thermogram or NMR spectra of PVA/geraniol NF. It was evident that the geraniol was not preserved in PVA NF without CD-IC owing to its volatile nature. On the other hand, enhanced thermal stability of geraniol was observed in the TGA thermogram of PVA/geraniol/ $\gamma$-CD-IC NF. Besides, NMR result of PVA/geraniol $/ \gamma$-CD-IC NF indicated that the initial $1: 1$ molar ratio of geraniol: $\gamma$-CD in geraniol $/ \gamma$-CD-IC did not change during the electrospinning process or after the storage. More importantly, the molar ratio of geraniol: $\gamma$-CD was found as 0.87:1 even after storage of these NF for two years at $\sim 22{ }^{\circ} \mathrm{C}$ and $\sim 25 \%$ relative humidity. Only $\sim 10 \%$ loss of geraniol was evaporated from PVA/geraniol/ $\gamma$-CD-IC NF confirming that the loss of geraniol was minimal after such a long storage period. The prolonged durability of geraniol was obviously observed owing to the $\mathrm{CD}$ inclusion complexation. PVA is a biodegradable synthetic polymer which is applicable in food packaging, and geraniol is widely used as fragrance/flavor having many specific properties such as antimicrobial and antioxidant. Hence, the electrospun PVA/ geraniol $/ \gamma$-CD-IC NF having very high surface area and nanoporous structure, as well as having geraniol with enhanced durability and thermal stability assisted by CD-IC may be quite applicable in functional food packaging and other food or medical related applications.

\section{Acknowledgments}

Dr. T. Uyar acknowledges The Scientific and Technological Research Council of Turkey (TUBITAK) (Project \# 111M459) and EU FP7-PEOPLE2009-RG Marie Curie-IRG (NANOWEB, PIRG06-GA-2009-256428) and Outstanding Young Scientists Award Program (TUBA-GEBIP) for funding the research. F. Kayaci acknowledges TUBITAK-BIDEB (Grant \# 2211) for the national Ph.D. student scholarship.

\section{References}

Allen, F. H. (2002). The Cambridge Structural Database: A quarter of a million crystal structures and rising. Acta Crystallographica, Section B: Structural Science, 58(3), 380-388. 
Bhardwaj, N., \& Kundu, S.C. (2010). Electrospinning: A fascinating fiber fabrication technique. Biotechnology Advances, 28(3), 325-347.

Blöchl, P. E. (1994). Projector augmented-wave method. Physical Review B, 50(24), 17953

Chen, W., \& Viljoen, A. (2010). Geraniol-A review of a commercially important fragrance material. South African Journal of Botany, 76(4), 643-651.

Ciobanu, A., Landy, D., \& Fourmentin, S. (2013). Complexation efficiency of cyclodextrins for volatile flavor compounds. Food Research International, 53(1), 110-114.

de Vos, P., Faas, M. M., Spasojevic, M., \& Sikkema, J. (2010). Encapsulation for preservation of functionality and targeted delivery of bioactive food components. International Dairy Journal, 20(4), 292-302.

Del Valle, E. M. (2004). Cyclodextrins and their uses: A review. Process Biochemistry, 39(9), 1033-1046.

DeMerlis, C., \& Schoneker, D. (2003). Review of the oral toxicity of polyvinyl alcoho (PVA). Food and Chemical Toxicology, 41(3), 319-326.

Fernandez, A., Torres-Giner, S., \& Lagaron, J. M. (2009). Novel route to stabilization of bioactive antioxidants by encapsulation in electrospun fibers of zein prolamine. Food Hydrocolloids, 23(5), 1427-1432.

Ge, L., Zhao, Y. -s., Mo, T., Li, J. -r., \& Li, P. (2012). Immobilization of glucose oxidase in electrospun nanofibrous membranes for food preservation. Food Control, 26(1), $188-193$.

Grimme, S. (2006). Semiempirical GGA-type density functional constructed with a longrange dispersion correction. Journal of Computational Chemistry, 27(15), 1787-1799.

Harata, K. (1987). The structure of the cyclodextrin complex. XX. Crystal structure of uncomplexed hydrated $\gamma$-cyclodextrin. Chemistry Society of Japan, 60, 2763-2767.

Hedges, A.R. (1998). Industrial applications of cyclodextrins. Chemical Reviews, 98(5), 2035-2044.

Hohenberg, P., \& Kohn, W. (1964). Inhomogeneous electron gas. Physical Review, 136(3B), B864.

Ignatova, M., Manolova, N., \& Rashkov, I. (2013). Electrospun antibacterial chitosan-based fibers. Macromolecular Bioscience, 13, 860-872.

Kant, A., Linforth, R. S. T., Hort, J., \& Taylor, A. J. (2004). Effect of $\beta$-cyclodextrin on aroma release and flavor perception. Journal of Agricultural and Food Chemistry, 52(7), 2028-2035.

Kayaci, F., Ertas, Y., \& Uyar, T. (2013). Enhanced thermal stability of eugenol by cyldextrin Inclusion complex encapsulated in electrospun polymeric nanofibers. Journal of Agricultural and Food Chemistry, 61(34), 8156-8165.

Kayaci, F., Umu, O. C., Tekinay, T., \& Uyar, T. (2013). Antibacterial electrospun poly(lactic acid) (PLA) nanofibrous webs incorporating triclosan/cyclodextrin inclusion complexes. Journal of Agricultural and Food Chemistry, 61(16), 3901-3908.

Kayaci, F., \& Uyar, T. (2011). Solid inclusion complexes of vanillin with cyclodextrins: their formation, characterization, and high-temperature stability. Journal of Agricultural and Food Chemistry, 59(21), 11772-11778.

Kayaci, F. \& Uyar, T. (2012). Encapsulation of vanillin/cyclodextrin inclusion complex in electrospun polyvinyl alcohol (PVA) nanowebs: Prolonged shelf-life and high temperature stability of vanillin. Food Chemistry, 133, 641-649.

Kohn, W., \& Sham, L. J. (1965). Self-consistent equations including exchange and correlation effects. Physical Review, 140(4A), A1133.

Kresse, G., \& Furthmüller, J. (1996a). Efficiency of ab-initio total energy calculations for metals and semiconductors using a plane-wave basis set. Computational Materials Science, 6(1), 15-50.

Kresse, G., \& Furthmüller, J. (1996b). Efficient iterative schemes for ab initio total-energy calculations using a plane-wave basis set. Physical Review B, 54(16), 11169.

Kriegel, C., Kit, K., McClements, D., \& Weiss, J. (2008). Nanofibers as carrier systems for antimicrobial microemulsions. Part I: Fabrication and characterization. Langmuir, 25(2), $1154-1161$.

Kuan, C. -Y., Yuen, K. -H., Bhat, R., \& Liong, M. -T. (2011). Physicochemical characterization of alkali treated fractions from corncob and wheat straw and the production of nanofibres. Food Research International, 44(9), 2822-2829.

Lapczynski, A., Bhatia, S., Foxenberg, R., Letizia, C., \& Api, A. (2008). Fragrance material review on geraniol. Food and Chemical Toxicology, 46(11), S160-S170.

Li, Y., Lim, L. T., \& Kakuda, Y. (2009). Electrospun zein fibers as carriers to stabilize (-)epigallocatechin gallate. Journal of Food Science, 74(3), C233-C240.

Lindner, K., \& Saenger, W. (1982). Crystal and molecular structure of cyclohepta-amylose dodecahydrate. Carbohydrate Research, 99(2), 103-115.

Manor, P. C., \& Saenger, W. (1974). Topography of cyclodextrin inclusion complexes. III. Crystal and molecular structure of cyclohexaamylose hexahydrate, the water dimer inclusion complex. Journal of the American Chemical Society, 96(11), 3630-3639.
Marcolino, V. A., Zanin, G. M., Durrant, L. R., Benassi, M.D. T., \& Matioli, G. (2011). Interaction of curcumin and bixin with $\beta$-cyclodextrin: complexation methods, stability, and applications in food. Journal of Agricultural and Food Chemistry, 59(7), 3348-3357.

Marques, H. M. C. (2010). A review on cyclodextrin encapsulation of essential oils and volatiles. Flavour and Fragrance Journal, 25(5), 313-326.

Mascheroni, E., Fuenmayor, C. A., Cosio, M. S., Di Silvestro, G., Piergiovanni, L., Mannino, S., et al. (2013). Encapsulation of volatiles in nanofibrous polysaccharide membranes for humidity-triggered release. Carbohydrate Polymers, 98(1), 17-25.

Neo, Y. P., Ray, S., Jin, J., Gizdavic-Nikolaidis, M., Nieuwoudt, M. K., Liu, D., et al. (2012). Encapsulation of food grade antioxidant in natural biopolymer by electrospinning technique: A physicochemical study based on zein-gallic acid system. Food Chemistry, 136(2), 1013-1021.

Perdew, J. P., Chevary, J., Vosko, S., Jackson, K. A., Pederson, M. R., Singh, D., et al. (1992). Atoms, molecules, solids, and surfaces: Applications of the generalized gradient approximation for exchange and correlation. Physical Review B, 46(11), 6671.

Puliti, R., Mattia, C. A., \& Paduano, L. (1998). Crystal structure of a new $\alpha$-cyclodextrin hydrate form. Molecular geometry and packing features: Disordered solvent contribution. Carbohydrate Research, 310(1), 1-8.

Ramakrishna, S. (2005). An introduction to electrospinning and nanofibers. Singapore: World Scientific Publishing.

Rusa, C. C., Bullions, T. A., Fox, J., Porbeni, F. E., Wang, X., \& Tonelli, A. E. (2002). Inclusion compound formation with a new columnar cyclodextrin host. Langmuir, 18(25), 10016-10023.

Saenger, W., Jacob, J., Gessler, K., Steiner, T., Hoffmann, D., Sanbe, H., et al. (1998). Structures of the common cyclodextrins and their larger analogues-Beyond the doughnut. Chemical Reviews, 98, 1787-1802.

Samperio, C., Boyer, R., Eigel, W. N., III, Holland, K. W., McKinney, J. S., O'Keefe, et al. (2010). Enhancement of plant essential oils' aqueous solubility and stability using alpha and beta cyclodextrin. Journal of Agricultural and Food Chemistry, 58(24), 12950-12956.

Snor, W., Liedl, E., Weiss-Greiler, P., Viernstein, H., \& Wolschann, P. (2009). Density functional calculations on meloxicam- $\beta$-cyclodextrin inclusion complexes. International Journal of Pharmaceutics, 381(2), 146-152.

Solórzano-Santos, F., \& Miranda-Novales, M. G. (2012). Essential oils from aromatic herbs as antimicrobial agents. Current Opinion in Biotechnology, 23(2), 136-141.

Stijnman, A.C., Bodnar, I., \& Hans Tromp, R. (2011). Electrospinning of food-grade polysaccharides. Food Hydrocolloids, 25(5), 1393-1398.

Szejtli, J. (1998). Introduction and general overview of cyclodextrin chemistry. Chemical Reviews, 98(5), 1743-1754.

Torres-Giner, S. (2011). Electrospun nanofibers for food packaging applications. In J. M. Lagaron (Ed.), Multifunctional and nanoreinforced polymers for food packaging. Woodhead Pub.

Torres-Giner, S., Gimenez, E., \& Lagaron, J. (2008). Characterization of the morphology and thermal properties of zein prolamine nanostructures obtained by electrospinning. Food Hydrocolloids, 22(4), 601-614.

Tsai, Y., Tsai, H. H., Wu, C. P., \& Tsai, F. J. (2010). Preparation, characterisation and activity of the inclusion complex of paeonol with $\beta$-cyclodextrin. Food Chemistry, 120(3), 837-841.

Uyar, T., Hacaloglu, J., \& Besenbacher, F. (2009). Electrospun polystyrene fibers containing high temperature stable volatile fragrance/flavor facilitated by cyclodextrin inclusion complexes. Reactive and Functional Polymers, 69(3), 145-150.

Uyar, T., Hacaloglu, J., \& Besenbacher, F. (2011). Electrospun polyethylene oxide (PEO) nanofibers containing cyclodextrin inclusion complex. Journal of Nanoscience and Nanotechnology, 11(5), 3949-3958.

Uyar, T., Hunt, M.A., Gracz, H. S., \& Tonelli, A. E. (2006). Crystalline cyclodextrin inclusion compounds formed with aromatic guests: Guest-dependent stoichiometries and hydration-sensitive crystal structures. Crystal Growth E Design, 6(5), 1113-1119.

Uyar, T., Nur, Y., Hacaloglu, J., \& Besenbacher, F. (2009). Electrospinning of functional poly (methyl methacrylate) nanofibers containing cyclodextrin-menthol inclusion complexes. Nanotechnology, 20(12), 125703

Vega-Lugo, A.C., \& Lim, L. T. (2009). Controlled release of allyl isothiocyanate using soy protein and poly (lactic acid) electrospun fibers. Food Research International, 42(8), 933-940.

Wendorff, J. H., Agarwal, S., \& Greiner, A. (2012). Electrospinning: Materials, processing and applications. John Wiley \&Sons Publishing. 